\title{
Incidence and patterns of recurrence of Ovarian Cancer in India; A study from Cancer Center in Northern India
}

\author{
Dr. Vineet Talwar*, Medical Oncology, Rajiv Gandhi Cancer Institute \& Research \\ Center, New Delhi, India
}

\section{Objectives}

To calculate the disease-free survival (DFS) and overall survival (OS) in ovarian cancer patients with non-metastatic disease and stage IV disease with pleural effusion only.

To assess the paclitaxel/ carboplatin related toxicity profile in study group cases.

\section{Methods}

Medical records of total 620 cases of ovarian cancer from Rajiv Gandhi Cancer Institute and Research Centre, Delhi during the years 2012-2013 were screened. 118 cases were selected as per the inclusion and exclusion criteria. The detailed data regarding patients' clinical history, tumor characteristics, therapy, and recurrence etc. were assembled from their clinical records. The statistical analyses were performed using IBM SPSS Statistics for Windows, Version 23.0.

\section{Results}

The mean and median ages at diagnosis were 49.1 and 50 (range 23-75) years respectively. The mean and median serum CA 125 levels were 1423 and 544 (range 5.5-12431) ng/ml. The family history of breast/ ovarian cancer was positive in $6.8 \%$ cases. In our study group, the majority of patients $(74.6 \%)$ were diagnosed at stage III. The histopathological evaluation revealed a large proportion of patients with high grade tumors (84.7\%) and serous adenocarcinoma (69.5\%) as primary histology morphology. Other than alopecia in all, $37.3 \%$ cases had experienced other toxicities due to chemotherapy. A proportion of 64.4 cases had experienced the recurrence of disease. The mean DFS and OS were 31.03 and 40.14 months.

\section{Conclusion}

Ovarian cancer is the fourth commonest cancer amongst females in Indian population. Most of the cases are detected in late stages, and have high grade tumor Histopathology, with frequent relapses. Further studies are ongoing to see the HBOC subset of patients.
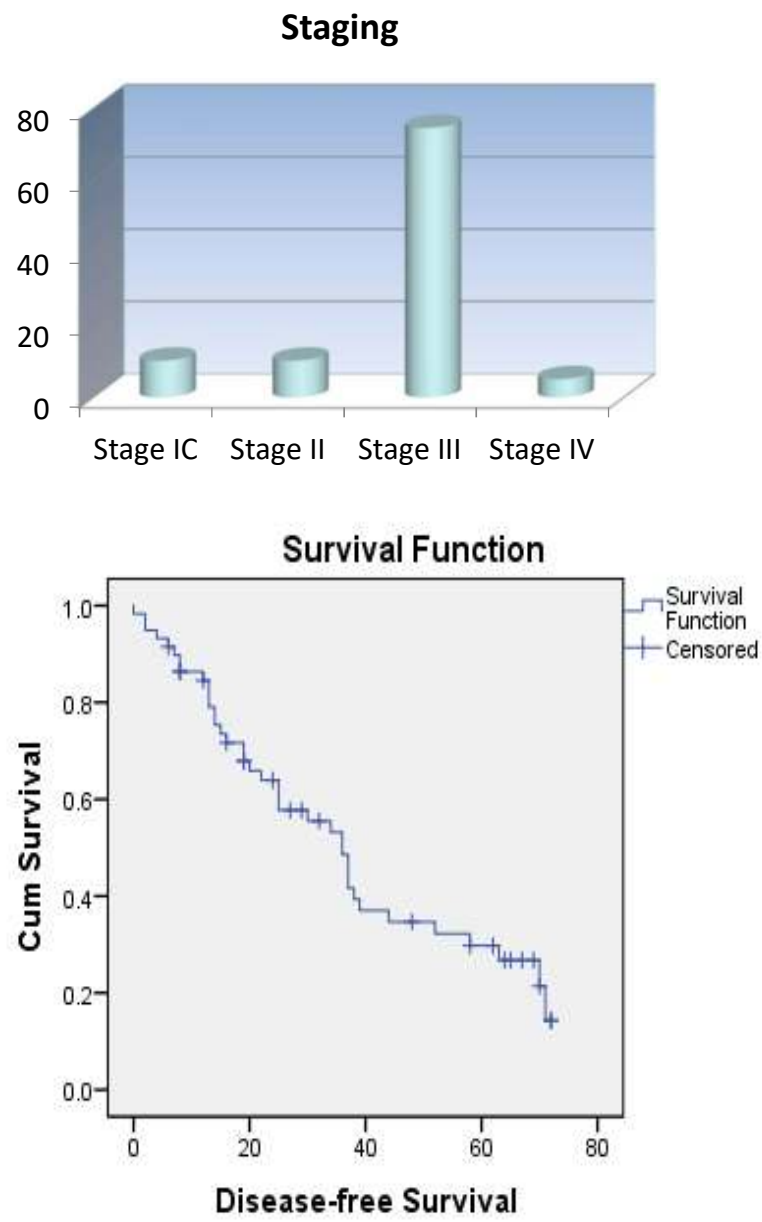